\title{
Modelling transport in nanoparticle organic solar cells using Monte Carlo methods
}

\author{
K. Feron, ${ }^{1,2, a)}$ S. Ulum, ${ }^{2}$ N. P. Holmes, ${ }^{2}$ A. L. D. Kilcoyne,${ }^{3}$ W. J. Belcher, ${ }^{2}$ X. Zhou, ${ }^{2}$ \\ C. J. Fell, ${ }^{1,2}$ and P. C. Dastoor ${ }^{2}$ \\ ${ }^{1}$ CSIRO Energy Technology, Newcastle, NSW 2300, Australia \\ ${ }^{2}$ Centre for Organic Electronics, University of Newcastle, Newcastle, NSW 2308, Australia \\ ${ }^{3}$ Advanced Light Source, Lawrence Berkeley National Laboratory, Berkeley, California 94720, USA
}

(Received 13 August 2013; accepted 21 October 2013; published online 5 November 2013)

\begin{abstract}
Water-based nanoparticle (NP) organic solar cells eliminate the need for harmful organic solvents during deposition. However, the core-shell NP structure should limit charge extraction resulting in poor performance. Here, we use dynamic Monte Carlo modelling to show that for optimised NP structures the core-shell character does not severely limit performance. Simulations further reveal that small NPs are more susceptible to extensive phase segregation, which diminishes charge carrier percolation pathways from the cores to the electrodes and thus inhibits charge extraction. Simulated performance behaviour was used to propose an explanation for the experimentally observed change in performance due to annealing. (C) 2013 AIP Publishing LLC. [http://dx.doi.org/10.1063/1.4829152]
\end{abstract}

Aqueous nanoparticle (NP) morphologies offer the prospect of direct control of domain size whilst simultaneously eliminating the need for hazardous organic solvents during the device fabrication process. ${ }^{1-4}$ As such, nanoparticle based organic photovoltaics (NP-OPVs) have considerable advantages over standard bulk heterojunction (BHJ) devices in terms of environmental, health, and safety issues. ${ }^{5,6}$ The NPs typically adopt a core-shell morphology, ${ }^{4,7,8}$ which for poly(3-hexylthiophene) (P3HT):[6,6]-phenyl-C61-buteric acid methylester (PCBM) core-shell structures, consists of an almost pure PCBM core and a P3HT rich (roughly 70\%) shell. ${ }^{7}$ This P3HT rich (and hence PCBM depleted) shell should limit electron extraction due to reduced coreelectrode charge carrier pathways. However, P3HT:PCBM NP-OPVs have been fabricated with efficiencies similar to those of the corresponding unannealed BHJ devices. ${ }^{7}$ These strides in efficiency were achieved by carefully controlling and optimising the nanostructure. However, a specific theoretical investigation of the core-shell structure and its impact on photovoltaic (PV) performance is currently lacking.

Dynamic Monte Carlo (DMC) models are a powerful tool for analysing morphological effects upon exciton generation and charge transport in OPVs. Indeed, BHJ, ${ }^{9-12}$ columnar, ${ }^{9,11,12}$ bilayer, ${ }^{11,12}$ and gyroid ${ }^{12}$ morphologies have all been investigated using DMC models. Furthermore, DMC models can quantitatively describe OPVs ${ }^{13}$ including standard P3HT:PCBM BHJ solar cells. ${ }^{14}$ In this letter, a DMC investigation of core-shell structures is presented. Internal quantum efficiency (IQE) and short-circuit current $\left(J_{s c}\right)$ are modelled for both NP and BHJ morphologies. Finally, DMC modelling is used to explain experimentally measured results.

The DMC model has been described previously. ${ }^{14,15}$ In short, the morphology was a discretized 3D lattice consisting of $1 \times 1 \times 1 \mathrm{~nm}$ voxels. Each voxel represents either electron

\footnotetext{
${ }^{\text {a) }}$ Author to whom correspondence should be addressed. Electronic mail: Krishna.Feron@csiro.au
}

donor material or electron acceptor material (see supplementary material for the corresponding energy levels ${ }^{22}$ ). BHJ morphologies were constructed using the cellular automata method described previously. ${ }^{15,16}$ Excitons are created in the system according to the optical field, which was assumed to be uniform throughout the active layer. This simplification negates effects due to the different optical properties of the $\mathrm{NP}$ and BHJ structures and thus allowed us to isolate morphological effects. Exciton hopping transport was modelled using Föster Resonance Energy Transfer theory taking into account energetic disorder. Following exciton dissociation, charge carrier hopping behaviour was modelled using Marcus theory; taking into account energetic disorder, the internal electric field set up by the work function difference of the electrodes, Coulomb interaction, and image charge induced at the electrodes. Adjacent charge carriers of opposite polarity are allowed to recombine with a fixed charge carrier recombination rate. Charge extraction at the electrode interface is also modelled using Marcus theory. In the DMC model, charge carriers are confined to their associated moieties. Even though a general analysis, which is not specific to any material system, of the core-shell structure is provided, the parameters used in the model are largely tuned towards describing P3HT:PCBM devices (see supplementary material $^{22}$ ). Performance parameters were determined when the system was in steady state, i.e., when the charge carrier population did not significantly change any more. DMC simulations were run 5 times for each configuration and the average of the performance parameters was determined.

Scanning transmission X-ray microscopy (STXM) measurements were conducted at the Advanced Light Source on beamline 5.3.2.2 (Ref. 17) and were used to obtain P3HT and PCBM composition maps as described previously., ${ }^{4,18}$ The P3HT:PCBM ratio of the virtual morphologies was 1:1; consistent with the P3HT:PCBM ratio used in device fabrication. ${ }^{7,19}$ A core-shell structure was obtained by placing a spherical core amidst a random P3HT:PCBM mixture, which 


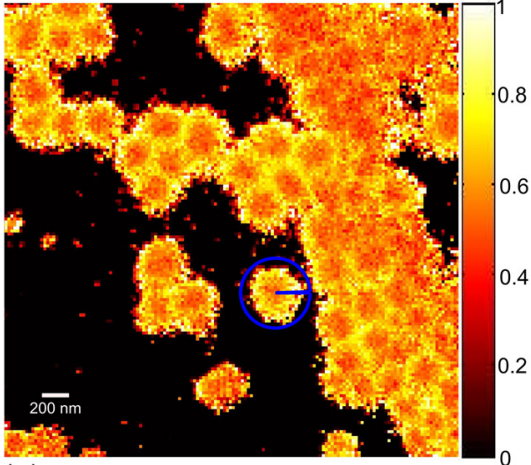

(a)

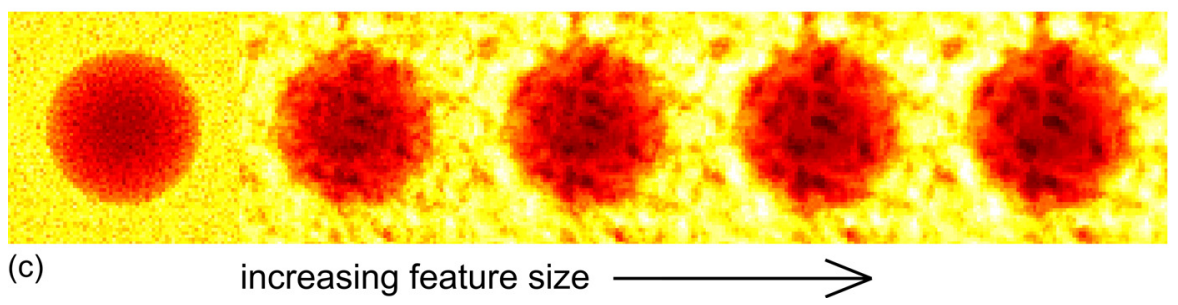

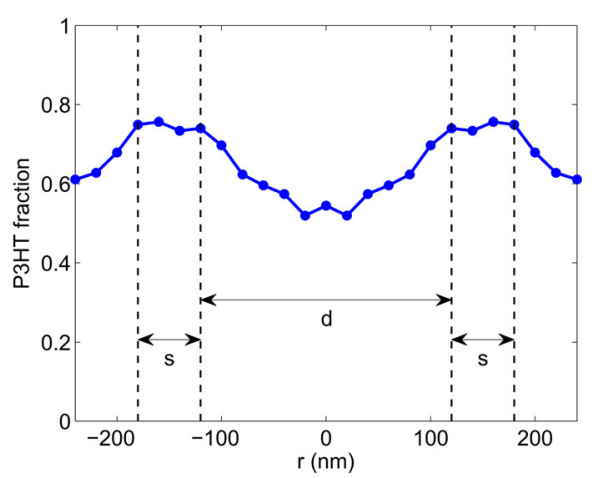

(b)

FIG. 1. (a) STXM P3HT composition map of P3HT:PCBM nanoparticles. The colours correspond to P3HT fraction as per the colour bar. Since these nanoparticles consist of P3HT and PCBM only, the PCBM composition maps (not shown) are the inverse of the P3HT maps. (b) Radially averaged composition profile corresponding to the blue circle indicated in (a). $d$ indicates the diameter of the core and $s$ the width of the shell. (c) P3HT composition map of a virtual nanoparticle as a function of feature size. The virtual nanoparticles have the following feature sizes, from left to right: $3.1 \mathrm{~nm}$, $6.2 \mathrm{~nm}, 8.8 \mathrm{~nm}, 12.1 \mathrm{~nm}$, and $14.2 \mathrm{~nm}$. The same colour scale is used as in (a). corresponds with the STXM composition maps (Figure 1(a)). Consistent with previous studies, the core was set to be pure PCBM. ${ }^{4,7}$ STXM measurements show that the shell composition consists predominantly of $\mathrm{P} 3 \mathrm{HT},{ }^{7}$ but varies between $65 \%$ and $76 \% .^{7,19}$ In order to evaluate the most limiting case of the core-shell structure, we chose a concentration of $75 \%$ for P3HT in the shell, which is at the upper limit of experimental observation. The core-shell structure can be spatially defined (Figure 1(b)) by the diameter of the cores, $d$, and half the distance between adjacent cores, $s$, which is the shell thickness. The NP size as measured using dynamic light scattering (described previously ${ }^{4,19,20}$ ) consists of both the diameter of the core and width of the shell, i.e., NP size $=d+2 s$. We investigated morphologies with $d: s$ ratios of 4 and 10, since particles with these $d: s$ ratios were observed in the measured composition maps. The $d: s$ ratio was seen to vary between roughly 3 and 10 . The feature size $(\phi)$, which is defined as

$$
\phi=\frac{3 V}{A}
$$

(where $V$ is the volume and $A$ is the heterointerface area), considerably affects exciton dissociation, ${ }^{15}$ charge recombination, ${ }^{11}$ and thus IQE. ${ }^{9}$ As such, the same cellular automata algorithm that was used to create the BHJ morphologies was also applied to the core-shell structures. This blend coarsening algorithm decreases the number of disconnected volumes, while still maintaining the core-shell character of the structure (Figure 1(c)). However, the P3HT shell concentration was observed to increase. The largest average deviation (12\%) of the composition profiles before and after the swapping procedure occurred for the smallest NP size with the largest feature size. The relationship between thickness and NP size relation indicates that NP films consist of three NP layers (see supplementary material ${ }^{22}$ ). Hence, virtual morphologies were constructed by stacking three NPs on top each other. For simplicity and computational efficiency, we assume that the nanoparticle layers stack in a primitive cubic fashion, i.e., an NP size of $30 \mathrm{~nm}$ results in an active layer thickness of $90 \mathrm{~nm}$. The $\mathrm{x}$ - and $\mathrm{y}$-dimensions of the lattice
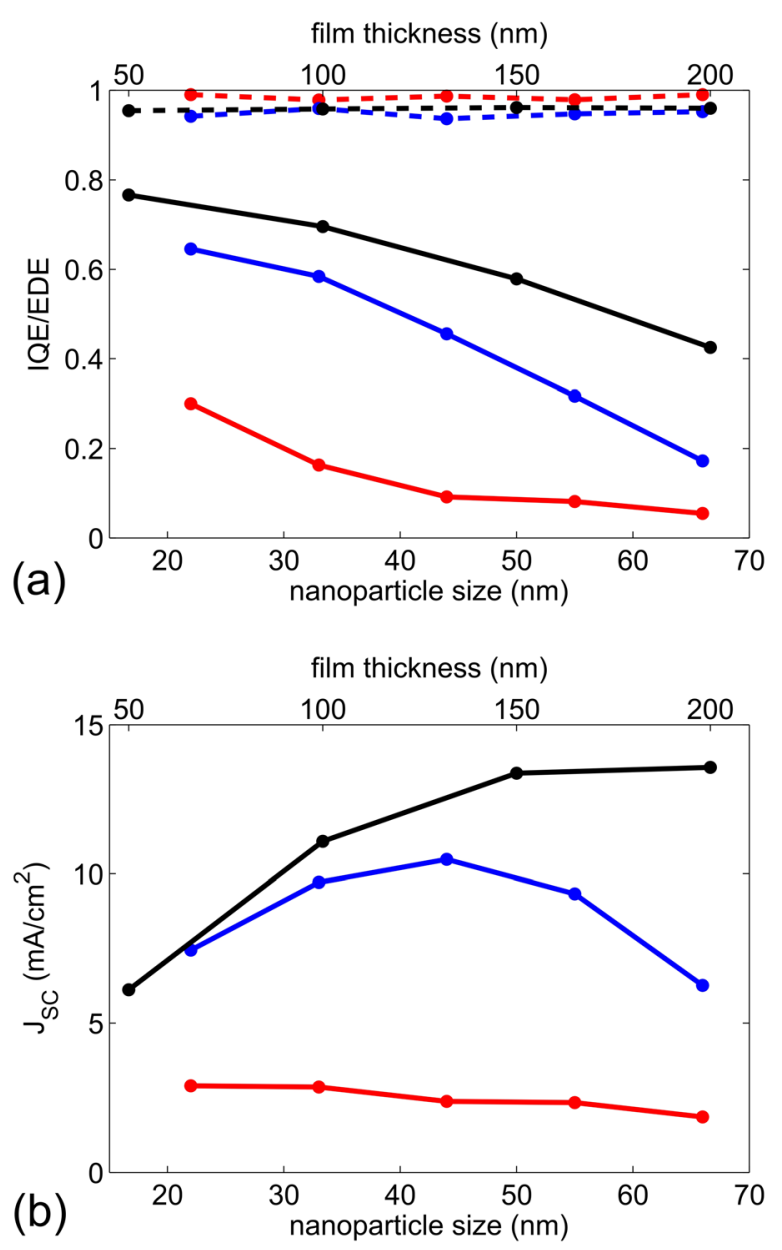

FIG. 2. (a) IQE (solid lines) and EDE (dashed lines) are shown as a function of NP size and film thickness for a BHJ morphology with a feature size of $14.2 \mathrm{~nm}$ (black) as well as core-shell morphologies with a feature size of $14.1 \mathrm{~nm}$ (blue) and $3.6 \mathrm{~nm}$ (red). (b) shows the corresponding $J_{S C}$. 


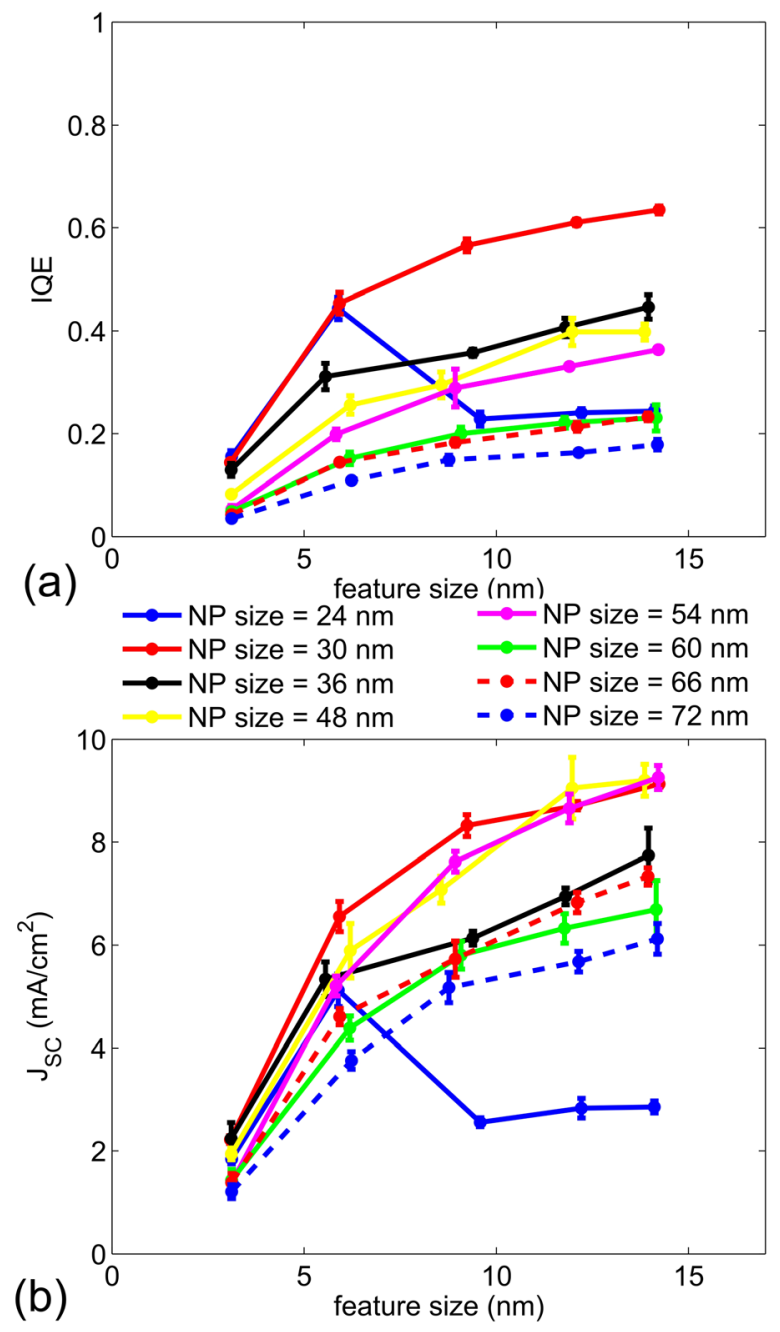

FIG. 3. (a) IQE and (b) $J_{S C}$ as a function of feature size for core-shell morphologies with a $d: s$ ratio of 4 .

were twice the NP size so that each simulated structure contained 12 NPs. However, periodic boundaries were invoked in the $\mathrm{x}$ - and $\mathrm{y}$-directions, which means that the virtual devices have an infinite effective area.

Figure 2(a) shows IQE and exciton dissociation efficiency (EDE) as a function of NP size and thickness for a BHJ morphology with a feature size of $14.2 \mathrm{~nm}$ as well as core-shell structures with feature sizes of $14.1 \mathrm{~nm}$ (similar to a standard BHJ structure ${ }^{14}$ ) and $3.6 \mathrm{~nm}$. Both core-shell structures had a $d: s$ ratio of 10 . While exciton dissociation is most efficient for the structure with a small feature size,
EDE only marginally decreased with increasing feature size and was the same (to within the simulation standard deviation) for the BHJ and core-shell structure of similar feature size. Hence, in the case of core-shell and BHJ structures, EDE depends primarily upon the feature size and less on the specific nature of the structure. Since the photoluminescence is less for NP devices compared to BHJ devices, ${ }^{7}$ the feature size is likely to be smaller in the NP devices despite using the same annealing conditions as for the BHJ devices. Since little change in EDE is observed, the observed drop in IQE with thickness arises mainly from decreased charge extraction efficiency (increased charge recombination) for both the $\mathrm{BHJ}$ and core-shell structures. Indeed, the core-shell structure with the small feature size (and hence few charge carrier percolation pathways to the electrodes) exhibits low $J_{S C}$ (and hence poor charge extraction) irrespective of NP size. However, the core-shell structure with the larger feature size $(14.1 \mathrm{~nm})$ does exhibit a $J_{S C}$ that is strongly dependent on NP size. Although charge recombination increases with thickness, thicker layers generate more charge due to increased light absorption and hence $J_{S C}$ generally exhibits a maximum as a function of thickness. ${ }^{21}$ Figure 2(b) reveals that core-shell and BHJ structures with comparable feature size exhibit comparable IQE and $J_{S C}$ for film thicknesses below $140 \mathrm{~nm}$ (roughly $J_{S C}$ optimised thickness for the coreshell structure). The thickness regime below $140 \mathrm{~nm}$ is relevant as peak efficiency of P3HT:PCBM devices occurs at a film thickness of $85 \mathrm{~nm} .{ }^{14}$ Hence, the DMC results show that, for these morphologies and frequently used film thickness regime, the core-shell OPV devices approach the efficiency of the BHJ devices.

The simulations were repeated for a range of core-shell structures with a $d: s$ ratio of 4 . The corresponding IQE and $J_{S C}$ as a function of feature size are shown in Figure 3. As before, the EDE depends predominantly on feature size (for the chosen exciton diffusion lengths) and not on NP size. Consequently, variations in IQE reflect changes in charge recombination. In thin layers (and thus small NP size) IQE is generally higher (see Figure 2(a)), because charge carriers only need to travel a short distance to reach the electrodes.

In the case of the smallest NP size $(24 \mathrm{~nm})$, however, the IQE rapidly drops for feature sizes above $6 \mathrm{~nm}$, resulting in a maximum IQE for an NP size of $30 \mathrm{~nm}$. The reason for this unexpected drop in IQE can be found in the corresponding composition maps (Figure 4). Initially, the cores are immersed in a mixture of P3HT and PCBM, but as feature

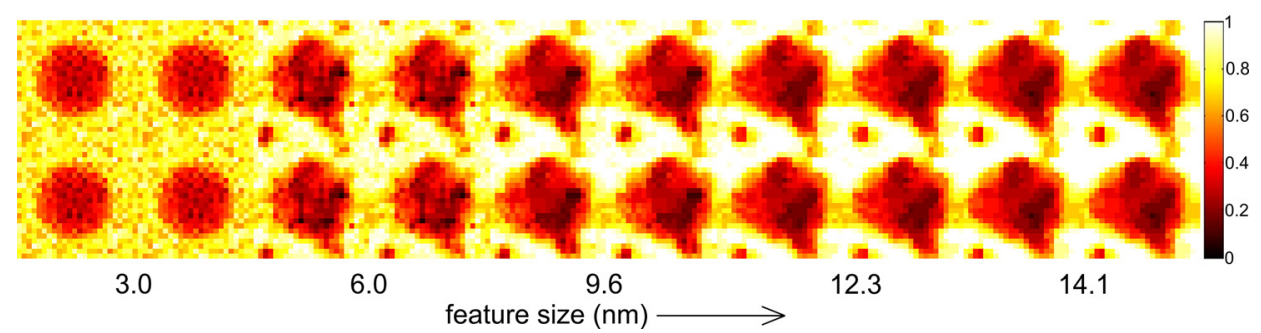

FIG. 4. Composition maps of core-shell structures with a nominal NP size of $24 \mathrm{~nm}$ and $d: s$ ratio of 4 . Feature size increases (from left to right) according to a cellular automata algorithm. The colours indicate P3HT mass composition as per the colour bar. Since these morphologies consist of P3HT and PCBM only, the PCBM composition maps (not shown) are the exact inverse of the P3HT maps. 
size increases the components phase segregate and, for feature sizes larger than $6 \mathrm{~nm}$, the mixed region disappears and pure domains of either PCBM or P3HT are formed. For the $24 \mathrm{~nm}$ NPs, extensive phase segregation occurs and thus percolation pathways from the PCBM cores to the electrodes are greatly reduced. These DMC simulations are in excellent agreement with recent experimental observations of gross phase segregation in NP devices correlated to poor device performance $^{7,19}$ and confirm that electron extraction is inhibited as a result of extensive phase segregation.

This extensive phase segregation was only observed for the smallest NP size; structures with larger NPs retained the mixed region and looked similar to the series shown in Figure 1(c) up to the maximum feature size investigated $(14.4 \mathrm{~nm}$ ). Hence, the highest IQE was achieved for the smallest NPs (i.e., thin layers) that do not exhibit extensive phase segregation, but still have a large feature size (i.e., $14.2 \mathrm{~nm}$ ).

As a result of the trade-off between IQE and light absorption with increasing thickness, virtual devices with a NP size between 30 and $54 \mathrm{~nm}$ exhibit similar high $J_{S C}$ values (Figure 3(b)). This size range is only indicative since the precise range of NP sizes that will exhibit high $J_{S C}$ will depend on the details of the spatial distribution of the optical field, which was assumed constant for this study. However, this result does reveal that real NP devices containing a range of NP sizes ${ }^{4,8}$ can generate broadly similar high $J_{S C}$ values.

Real P3HT:PCBM NP devices with varying NP size were fabricated and I-V curves were measured before and after annealing. Annealing conditions $\left(140^{\circ} \mathrm{C}\right.$ for $\left.4 \mathrm{~min}\right)$ were optimised for efficiency as shown in the supplementary material. ${ }^{22}$ Our device fabrication procedure has been described previously. ${ }^{7}$ Device characterisation was achieved using J-V curve measurements as described previously. ${ }^{4,19,20}$ The highest measured values of $J_{S C}$ and power conversion efficiency $(\eta)$ were at the NP size of $36 \mathrm{~nm}$ for pristine devices and $33 \mathrm{~nm}$ for annealed devices (Figure 5, inset), which correlates well with the optimum predicted by DMC simulations

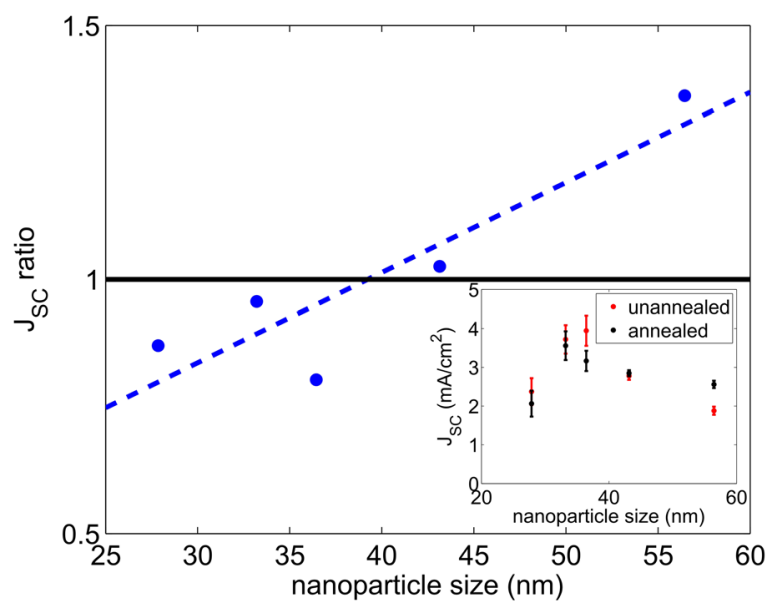

FIG. 5. The ratio of $J_{S C}$ after and before annealing as a function of NP size. The linear fit (dashed line) serves as a guide to the eye. The inset shows the $J_{S C}$ of real NP devices before and after annealing. $J_{S C}$ was averaged over 6 different devices for each NP size with the corresponding error bars (standard deviation) shown. for the NPs with a $d: s$ ratio of 4 . The simulated structures with a d:s ratio of 10 exhibited a maximum at $45 \mathrm{~nm}$. Consequently, real devices are likely to be dominated by structures with a d:s ratio close to 4 , i.e., core-shell structures with a relatively thick shell. Figure 5 reveals that $J_{S C}$ decreases after annealing for NP sizes less than $42 \mathrm{~nm}$. In light of the DMC results discussed in this letter, annealing these smaller NPs produces gross phase segregation, which inhibits electron extraction. By comparison, for devices with larger NPs, mixed P3HT:PCBM regions with electron pathways to the electrodes are likely to be retained (and improved) after annealing resulting in the observed increase in $J_{S C}$.

In conclusion, DMC simulations show that structures with a core-shell character are not severely limited by charge recombination when optimised for NP size and feature size. In fact, similar IQE and $J_{S C}$ as BHJ structures are predicted for optimised core-shell structures; demonstrating that the NP approach, which allows for printing from water as opposed to harmful organic solvents, is a viable alternative to standard BHJ fabrication. The DMC simulations reveal that small NPs are more susceptible to extensive phase segregation, which diminishes charge carrier percolation pathways from the cores to the electrodes and thus inhibits charge extraction. On the other hand, a structure characterised by a very small feature size may also have limited charge carrier pathways to the electrodes. Hence, it is crucial to optimise annealing conditions to prevent severe charge recombination. A real NP device contains NPs with a range of sizes. Optimum annealing conditions depend on NP size and cannot be assumed to be the same across a range of NP sizes. DMC simulations predict high $J_{S C}$ values for an approximately $25 \mathrm{~nm}$ range of NP sizes. Real NP devices were fabricated and the empirically determined optimum NP size correlates well with DMC results suggesting that the devices are dominated by NPs with a relatively thick shell. Finally, the phase segregation behaviour seen in DMC simulations was used to propose an explanation for the experimentally observed change in $J_{S C}$ due to annealing.

This work was supported by the Australian Government, through the Australian Renewable Energy Agency. The University of Newcastle and CSIRO Energy Technology are gratefully acknowledged for a $\mathrm{PhD}$ scholarship (KF). The Australian Solar Institute is acknowledged for a PhD scholarship (NH). The Indonesian Directorate General of Higher Education (DIKTI) is acknowledged for PhD scholarship funding (SU). We acknowledge financial support from the Commonwealth of Australia through the Access to Major Research Facilities Program. The ALS is supported by the Director, Office of Science, Office of Basic Energy Sciences, of the U.S. Department of Energy under Contract No. DE-AC02-05CH11231. In addition, we acknowledge Kerry Burke for his contribution in obtaining and analysing the STXM composition profiles. This work was performed in part at the Materials node of the Australian National Fabrication Facility. A company established under the National Collaborative Research Infrastructure Strategy to provide nano and microfabrication facilities for Australia's researchers. 
${ }^{1}$ K. Landfester, R. Montenegro, U. Scherf, R. Güntner, U. Asawapirom, S. Patil, D. Neher, and T. Kietzke, Adv. Mater. 14, 651 (2002).

${ }^{2}$ E. Hittinger, A. Kokil, and C. Weder, Angew. Chem., Int. Ed. 43, 1808 (2004).

${ }^{3}$ H. J. Snaith and R. H. Friend, Synth. Met. 147, 105 (2004).

${ }^{4}$ K. B. Burke, A. J. Stapleton, B. Vaughan, X. Zhou, A. L. D. Kilcoyne, W. J. Belcher, and P. C. Dastoor, Nanotechnology 22, 265710 (2011).

${ }^{5}$ E. M. Ward, P. A. Schulte, K. Straif, N. B. Hopf, J. C. Caldwell, T. Carreón, D. M. DeMarini, B. A. Fowler, B. D. Goldstein, K. Hemminki, C. J. Hines, K. H. Pursiainen, E. Kuempel, J. Lewtas, R. M. Lunn, E. Lynge, D. M. McElvenny, H. Muhle, T. Nakajima, L. W. Robertson, N. Rothman, A. M. Ruder, M. K. Schubauer-Berigan, J. Siemiatycki, D. Silverman, M. T. Smith, T. Sorahan, K. Steenland, R. G. Stevens, P. Vineis, S. H. Zahm, L. Zeise, and V. J. Cogliano, Environ. Health Perspect. 118, 1355 (2010).

${ }^{6}$ A. M. Ruder, Ann. N. Y. Acad. Sci. 1076, 207 (2006).

${ }^{7}$ S. Ulum, N. Holmes, D. Darwis, K. Burke, A. L. D. Kilcoyne, X. Zhou, W. Belcher, and P. Dastoor, Sol. Energy Mater. Sol. Cells 110, 43 (2013).

${ }^{8}$ A. Stapleton, B. Vaughan, B. Xue, E. Sesa, K. Burke, X. Zhou, G. Bryant, O. Werzer, A. Nelson, A. L. D. Kilcoyne, L. Thomsen, E. Wanless, W. Belcher, and P. Dastoor, Sol. Energy Mater. Sol. Cells 102, 114 (2012).

${ }^{9}$ P. K. Watkins, A. B. Walker, and G. L. B. Verschoor, Nano Lett. 5, 1814 (2005).

${ }^{10}$ L. Meng, Y. Shang, Q. Li, Y. Li, X. Zhan, Z. Shuai, R. G. E. Kimber, and A. B. Walker, J. Phys. Chem. B 114, 36 (2010).

${ }^{11}$ R. A. Marsh, C. Groves, and N. C. Greenham, J. Appl. Phys. 101, 083509 (2007).
${ }^{12}$ R. G. E. Kimber, A. B. Walker, G. E. Schröder-Turk, and D. J. Cleaver, Phys. Chem. Chem. Phys. 12, 844 (2010).

${ }^{13}$ R. G. E. Kimber, E. N. Wright, S. E. J. O'Kane, A. B. Walker, and J. C. Blakesley, Phys. Rev. B 86, 235206 (2012).

${ }^{14}$ K. Feron, C. J. Fell, L. J. Rozanski, B. B. Gong, N. Nicolaidis, W. J. Belcher, X. Zhou, E. Sesa, B. V. King, and P. C. Dastoor, Appl. Phys. Lett. 101, 193306 (2012).

${ }^{15}$ K. Feron, X. Zhou, W. J. Belcher, and P. C. Dastoor, J. Appl. Phys. 111, 044510 (2012).

${ }^{16}$ P. Peumans, S. Uchida, and S. R. Forrest, Nature 425, 158 (2003).

${ }^{17}$ A. L. D. Kilcoyne, T. Tyliszczak, W. F. Steele, S. Fakra, P. Hitchcock, K. Franck, E. Anderson, B. Harteneck, E. G. Rightor, G. E. Mitchell, A. P. Hitchcock, L. Yang, T. Warwick, and H. Ade, J. Synchrotron Radiat. 10, 125 (2003).

${ }^{18}$ C. R. McNeill, B. Watts, L. Thomsen, W. J. Belcher, N. C. Greenham, and P. C. Dastoor, Nano Lett. 6, 1202 (2006).

${ }^{19}$ N. P. Holmes, K. B. Burke, P. Sista, M. Barr, H. D. Magurudeniya, M. C. Stefan, A. L. D. Kilcoyne, X. Zhou, P. C. Dastoor, and W. J. Belcher, Sol. Energy Mater. Sol. Cells 117, 437 (2013).

${ }^{20}$ S. Ulum, N. Holmes, M. Barr, A. L. D. Kilcoyne, B. B. Gong, X. Zhou, W. Belcher, and P. Dastoor, Nano Energy 2, 897 (2013).

${ }^{21}$ A. J. Moulé, J. B. Bonekamp, and K. Meerholz, J. Appl. Phys. 100, 094503 (2006).

${ }^{22}$ See supplementary material at http://dx.doi.org/10.1063/1.4829152 for the simulation parameters, the film thickness-NP size relationship and optimisation of annealing conditions. 